\title{
Serelaxin treatment does not reduce incidence of death in patients with AHF
}

In the RELAX-AHF trial published in 2013, patients with acute heart failure (AHF) treated with serelaxin, a recombinant form of human relaxin 2 , had a reduced incidence of worsening HF and cardiovascular death. However, this trial was not powered to assess effects on mortality. Investigators in the RELAX-AHF-2 trial, which is five times larger than the previous trial, now report that early administration of serelaxin in patients admitted to hospital for AHF did not have a significant effect on cardiovascular mortality.

Relaxin is a pleiotropic hormone with powerful anti-fibrotic and anti-inflammatory properties. Given the vasodilatory effects and possible cardioprotective effects of this hormone, serelaxin was developed as a potential therapy for the treatment of AHF.

The RELAX-AHF-2 study was a multicentre, randomized, event-driven trial designed to test whether serelaxin treatment reduced the incidence of death at 180 days or the incidence of worsening HF in the first 5 days compared with placebo. In total,
3,274 patients assigned to serelaxin treatment and 3,271 assigned to placebo were included in the analysis. At day 180, no significant difference was observed in death from cardiovascular causes between serelaxin-treated and placebo-treated patients (8.7\% versus $8.9 \%$; HR 0.98 , 95\% CI $0.83-1.15$, $P=0.77)$. In addition, at day 5 , no significant difference in worsening HF was observed between the two groups ( $6.9 \%$ versus $7.7 \%$; HR 0.89, 95\% CI 0.75-1.07, $P=0.19$ ).

To conclude, the results of the RELAX-AHF-2 trial did not replicate the benefit of serelaxin seen in the previous RELAX-AHF trial. "The negative result for the end point of death from cardiovascular causes in the RELAX-AHF- 2 trial is ... consistent with the conclusion that the result in the RELAX-AHF trial was a chance finding," explain the investigators.

Karina Huynh

ORIGINAL ARTICLE Metra, M. et al. Effects of serelaxin in patients with acute heart failure. N. Engl.J. Med. 381, 716-726 (2019)

\section{VALVULAR DISEASE}

\section{Still no benefit of MitraClip in MITRA-FR}

The 1-year results of the MITRA-FR trial reported in 2018 showed that the addition of percutaneous mitral valve repair to medical treatment did not improve outcomes compared with medical treatment alone in patients with heart failure and severe secondary mitral regurgitation (MR). These findings contrast with the COAPT trial results, reported shortly after, showing that mitral valve repair in addition to medical therapy improved outcomes in patients with heart failure and moderate-to-severe or severe secondary MR at 2 years. Many reasons were suggested for the different findings, including the longer follow-up in COAPT. However, the 2-year results of the MITRA-FR trial, presented at the ESC Congress 2019, confirm the 1-year findings.

The MITRA-FR trial was conducted at 37 centres in France and included 304 patients with symptomatic heart failure and severe secondary MR randomly assigned to percutaneous valve repair with a MitraClip device plus medical treatment or medical treatment alone. At 2 years, the rate of the primary efficacy outcome (all-cause death and unplanned hospitalization for heart failure) was similar in both groups $(63.8 \%$ versus $67.1 \%$; HR 1.01 , $95 \%$ Cl 0.77-1.34). Rates of all-cause death and hospitalization for heart failure were also similar in both groups. Interestingly, in the second year of follow-up, the MitraClip group had a lower rate of first hospitalization for heart failure. "This difference in the second year is even more visible for the cumulative number of hospitalizations, which was the primary end point of COAPT," says the MITRA-FR investigator Jean François Obadia, who nevertheless cautions that this analysis was not prespecified and explains that they will continue the follow-up to 5 years to assess whether the decrease in rehospitalizations is sustained. "Rather than contradictory, we consider the two studies complementary, and we are now planning with the COAPT team a meta-analysis on individual data from the two trials to better understand this complex and severe disease," he comments. Irene Fernández-Ruiz

ORIGINAL ARTICLE lung, B. et al. Percutaneous repair or medical treatment for secondary mitral regurgitation: outcomes at 2 years. Eur.J. Heart Fail. https://doi.org/10.1002/ ejhf.1616 (2019)

\section{PREVENTION}

\section{Use of polypills for CVD prevention}

Although fixed-dose combination (or polypill) therapy has been proposed as a cost-saving approach to improve drug adherence and reduce the risk of cardiovascular disease (CVD), the long-term effectiveness of such a strategy has not yet been established, particularly in primary prevention settings. Investigators in the Polylran study now report that a fixed-dose polypill strategy reduces the risk of cardiovascular events compared with non-pharmacological interventions.

The Polylran study was a two-group, pragmatic study nested within the Golestan Cohort Study, a large cohort study with 50,045 participants from the Golestan region of Iran. In Polylran, 3,421 individuals received a four-component polypill containing aspirin, atorvastatin, hydrochlorothiazide and either enalapril or valsartan, in addition to nonpharmacological intervention, which involved educational training about healthy lifestyle habits (polypill group), whereas 3,417 individuals received non-pharmacological intervention only (minimal-care group).

At the 5-year follow-up, $5.9 \%$ of individuals in the polypill group had major cardiovascular events compared with $8.8 \%$ of the minimalcare group (adjusted HR 0.66, 95\% Cl 0.55-0.80). This benefit was independent of whether the individual had pre-existing CVD. The reduction in risk of CVD was even more pronounced when comparing only those participants in the polypill group who had high adherence to the polypill with those in the minimal-care group (adjusted HR 0.43, $95 \% \mathrm{Cl} 0.33-0.55)$. Importantly, the rate of adverse events, such as intracranial haemorrhage, was not significantly different between the two treatment groups. Furthermore, in a post-hoc analysis, longer duration of polypill use was linked with a stronger protective effect.

"This pragmatic trial provides evidence that a polypill strategy could be considered as part of preventive programmes to reduce [CVD] burden among eligible adults, especially in [low-income and middle-income countries]," conclude the investigators.

Karina Huynh

ORIGINAL ARTICLE Roshandel, G. et al. Effectiveness of polypill for primary and secondary prevention of cardiovascular diseases (Polylran): a pragmatic,

cluster-randomised trial. Lancet 394, 672-683 (2019) RELATED ARTICLE Chow, C. K. \& Meng, Q. Polypills for primary prevention of cardiovascular disease. Nat. Rev. Cardiol. https://doi.org/10.1038/s41569-019-0209-y (2019) 